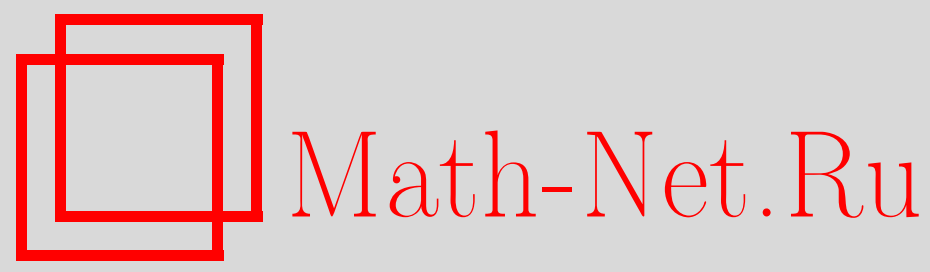

В. Н. Масленникова, Ю. С. Верещагина, Краевая задача для уравнения Пуассона для двух сред в $L_{p}^{2}$-пространствах, Матем. заметки, 1999, том 66, выпуск 4, 515-526

DOI: https://doi.org/10.4213/mzm1195

Использование Общероссийского математического портала Math-Net.Ru подразумевает, что вы прочитали и согласны с пользовательским соглашением http://www . mathnet.ru/rus/agreement

Параметры загрузки:

IP : 3.89 .197 .203

26 апреля 2023 г., 03:22:57 


\section{КРАЕВАЯ ЗАДАЧА ДЛЯ УРАВНЕНИЯ ПУАССОНА ДЛЯ ДВУХ СРЕД В $L_{p}^{2}$-ПРОСТРАНСТВАХ}

\section{В.Н. Масленникова, Ю. С. Верещагина}

В работе изучается краевая задача сопряжения для двух сред для уравнения Пуассона $\mu \Delta u=f(x)$ с решениями в классе $L_{p}^{2}\left(\mathbb{R}_{ \pm}^{3}\right), 1<p<\infty$, с соответствующей полунормой, где

$$
\mathbb{R}_{ \pm}^{3}=\left\{x \mid x^{\prime}=\left(x_{1}, x_{2}\right) \in \mathbb{R}^{2}, x_{3} \gtrless 0\right\}, \quad \mu= \begin{cases}\mu_{+}, & x_{3}>0 \\ \mu_{-}, & x_{3}<0 .\end{cases}
$$

Доказано существование решения для всех $f(x) \in L_{p}$ и получены априорные оценки решения с помощью мультипликаторов в пространстве $L_{p}^{2}\left(\mathbb{R}_{ \pm}^{3}\right)$. Найдено явное решение задачи для всех $f(x) \in \stackrel{\circ}{C}\left(\mathbb{R}^{3}\right)$. Построено (в явном виде) ядро оператора, порождаемого задачей, в виде полинома первой степени.

Библиограффия: 7 названий.

Планируя в будущем изучить задачу сопряжения для системы Стокса и Навье-Стокса, в том числе с неизвестной границей [1], [2], в данной работе мы изучаем задачу сопряжения для уравнения Пуассона для двух сред

$$
\mu \Delta u=f, \quad x \in \mathbb{R}_{+}^{3} \cup \mathbb{R}_{-}^{3},
$$

где

$$
\mathbb{R}_{ \pm}^{3}=\left\{x \mid x^{\prime}=\left(x_{1}, x_{2}\right) \in \mathbb{R}^{2}, x_{3} \gtrless 0\right\}, \quad \mu= \begin{cases}\mu_{+}, & x_{3}>0, \\ \mu_{-}, & x_{3}<0,\end{cases}
$$

$\mu_{+}, \mu_{-}-$положительные постоянные. Условия сопряжения на гранище раздела двух сред, т.е. на гиперплоскости $x_{3}=0$, имеют вид

$$
\begin{gathered}
\left.u\right|_{x_{3}=+0}=\left.u\right|_{x_{3}=-0}, \\
\left.\mu_{+} \frac{\partial u}{\partial n}\right|_{x_{3}=+0}=\left.\mu_{-} \frac{\partial u}{\partial n}\right|_{x_{3}=-0} .
\end{gathered}
$$

Задача (1)-(3) моделирует стационарное распределение температуры с разньми коэффициентами теплопроводности для двух сред. Решение исследуется в пространстве $u(x) \in L_{p}^{2}\left(\mathbb{R}_{+}^{3} \cup \mathbb{R}_{-}^{3}\right)$ (для краткости $\left.L_{p}^{2}\left(\mathbb{R}_{ \pm}^{3}\right)\right)$ с полунормой

$$
\|u\|_{L_{p}^{2}\left(\mathbb{R}_{ \pm}^{3}\right)}=\sum_{|\alpha|=2}\left\|D^{\alpha} u\right\|_{L_{p}\left(\mathbb{R}_{ \pm}\right)}, \quad 1<p<\infty, \quad f(x) \in L_{p}\left(\mathbb{R}^{3}\right) .
$$

Работа выполнена при финансовой поддержке Российского фонда фундаментальных исследований, грант № 99-01-01139. 
Так как область, где рассматривается решение, неограничена, иногда будет использоваться условие на бесконечности:

$$
\lim _{\left|x_{3}\right| \rightarrow \infty} u(x)=0
$$

В работе построено в явном виде решение задачи (1)-(4) для $f \in \stackrel{\circ}{C}^{\infty}\left(\mathbb{R}^{3}\right)$, получена оценка вторых производных решения в полунорме $L_{p}^{2}\left(\mathbb{R}_{ \pm}^{3}\right)$ :

$$
\sum_{|\alpha|=2}\left\|D_{x}^{\alpha} u\right\|_{L_{p}\left(\mathbb{R}_{+}^{3}\right)}+\sum_{|\alpha|=2}\left\|D_{x}^{\alpha} u\right\|_{L_{p}\left(\mathbb{R}_{-}^{3}\right)} \leqslant M\|f\|_{L_{p}\left(\mathbb{R}^{3}\right)}
$$

с постоянной $M>0$, не зависящей от $u(x)$, доказано сушествование решения задачи (1)-(3) для всех $f(x) \in L_{p}\left(\mathbb{R}^{3}\right)$. Доказано также, что размерность ядра оператоpa, порожденного задачей $(1)-(3)$, с областью определения $L_{p}^{2}\left(\mathbb{R}_{ \pm}^{3}\right)$ и областью значений $L_{p}\left(\mathbb{R}^{3}\right)$ равна 1 . При доказательстве результатов используется теория мультипликаторов преобразований Фурье [3], неравенства Харди [4], [5] и теория обобщенных функций $[6]$.

1. Построение явного решения. Построим решение задачи (1)-(4) для $f(x) \in$ $\stackrel{\circ}{C}^{\infty}\left(\mathbb{R}^{3}\right)$. Имеет место

Tеорема 1. Если $f(x) \in \stackrel{\circ}{C}^{\infty}\left(\mathbb{R}^{3}\right)$ и выполняется условие (4), то решение задачи (1)-(3) имеет вид $u\left(x^{\prime}, x_{3}\right)=F_{\xi^{\prime} \rightarrow x^{\prime}}^{-1}\left[\widehat{u}\left(\xi^{\prime}, x_{3}\right)\right]$, где

$$
\begin{aligned}
& \widehat{u}\left(\xi^{\prime}, x_{3}\right)=\frac{e^{-\left|\xi^{\prime}\right| x_{3}}}{\left(\mu_{-}-\mu_{+}\right)\left|\xi^{\prime}\right|} \int_{0}^{-\infty} \hat{f}\left(\xi^{\prime}, y_{3}\right) e^{\left|\xi^{\prime}\right| y_{3}} d y_{3} \\
& +\frac{e^{-\left|\xi^{\prime}\right| x_{3}}\left(\mu_{-}+\mu_{+}\right)}{2 \mu_{+}\left(\mu_{-}-\mu_{+}\right)\left|\xi^{\prime}\right|} \int_{0}^{+\infty} \hat{f}\left(\xi^{\prime}, y_{3}\right) e^{-\left|\xi^{\prime}\right| y_{3}} d y_{3} \\
& -\frac{e^{\left|\xi^{\prime}\right| x_{3}}}{2 \mu_{+}\left|\xi^{\prime}\right|} \int_{x_{3}}^{+\infty} \hat{f}\left(\xi^{\prime}, y_{3}\right) e^{-\left|\xi^{\prime}\right| y_{3}} d y_{3} \\
& -\frac{e^{-\left|\xi^{\prime}\right| x_{3}}}{2 \mu_{+}\left|\xi^{\prime}\right|} \int_{0}^{x_{3}} \hat{f}\left(\xi^{\prime}, y_{3}\right) e^{\left|\xi^{\prime}\right| y_{3}} d y_{3}, \quad x_{3}>0, \\
& \widehat{u}\left(\xi^{\prime}, x_{3}\right)=\frac{e^{\left|\xi^{\prime}\right| x_{3}}\left(\mu_{-}+\mu_{+}\right)}{2 \mu_{-}\left|\xi^{\prime}\right|\left(\mu_{-}-\mu_{+}\right)} \int_{0}^{-\infty} \hat{f}\left(\xi^{\prime}, y_{3}\right) e^{|\xi| y_{3}} d y_{3} \\
& +\frac{e^{\left|\xi^{\prime}\right| x_{3}}}{\left(\mu_{-}-\mu_{+}\right)\left|\xi^{\prime}\right|} \int_{0}^{+\infty} \hat{f}\left(\xi^{\prime}, y_{3}\right) e^{-\left|\xi^{\prime}\right| y_{3}} d y_{3} \\
& -\frac{e^{-\left|\xi^{\prime}\right| x_{3}}}{2 \mu_{-}\left|\xi^{\prime}\right|} \int_{-\infty}^{x_{3}} \hat{f}\left(\xi^{\prime}, y_{3}\right) e^{\left|\xi^{\prime}\right| y_{3}} d y_{3} \\
& +\frac{e^{\left|\xi^{\prime}\right| x_{3}}}{2 \mu_{-}\left|\xi^{\prime}\right|} \int_{0}^{x_{3}} \hat{f}\left(\xi^{\prime}, y_{3}\right) e^{-\left|\xi^{\prime}\right| y_{3}} d y_{3}, \quad x_{3}<0
\end{aligned}
$$


ДокАЗАТЕльСтво. Применяя к (1) преобразование Фурье по $x^{\prime}=\left(x_{1}, x_{2}\right)$, получаeм

$$
\frac{\partial^{2} \widehat{u}}{\partial x_{3}^{2}}-\left|\xi^{\prime}\right|^{2} \widehat{u}=\frac{\hat{f}\left(\xi^{\prime}, x_{3}\right)}{\mu}
$$

Общее решение соответствующего (7) однородного уравнения имеет вид

$$
\widehat{u}_{0}\left(\xi^{\prime}, x_{3}\right)=a\left(\xi^{\prime}\right) e^{-\left|\xi^{\prime}\right| x_{3}}+b\left(\xi^{\prime}\right) e^{\left|\xi^{\prime}\right| x_{3}} .
$$

Найдем частное решение уравнения (7). Рассмотрим задачу Коши

$$
\begin{aligned}
& \frac{\partial^{2} \widetilde{u}}{\partial x_{3}^{2}}-\left|\xi^{\prime}\right|^{2} \widetilde{u}=\frac{\hat{f}\left(\xi^{\prime}, x_{3}\right)}{\mu}, \\
& \widetilde{u}\left(\xi^{\prime}, 0\right)=\widetilde{u}_{x_{3}}^{\prime}\left(\xi^{\prime}, 0\right)=0 .
\end{aligned}
$$

Ее решением является

$$
\widetilde{u}\left(\xi^{\prime}, x_{3}\right)=\int_{0}^{x_{3}} \varphi\left(\xi^{\prime}, x_{3}-y_{3}\right) \frac{\hat{f}\left(\xi^{\prime}, y_{3}\right)}{\mu} d y_{3},
$$

где $\varphi\left(\xi^{\prime}, x_{3}\right)$ - решение задачи

$$
\varphi_{x_{3}}^{\prime \prime}-\left|\xi^{\prime}\right|^{2} \varphi=0, \quad \varphi\left(\xi^{\prime}, 0\right)=0, \quad \varphi_{x_{3}}^{\prime}\left(\xi^{\prime}, 0\right)=1,
$$

т.е. $\varphi\left(\xi^{\prime}, x_{3}\right)=c_{1} \operatorname{ch}\left(\left|\xi^{\prime}\right| x_{3}\right)+c_{2} \operatorname{sh}\left(\left|\xi^{\prime}\right| x_{3}\right)$. Постоянные $c_{1}, c_{2}$ определяем из начальных условий: $c_{1}=0, c_{2}=1 /\left|\xi^{\prime}\right|$; откуда

$$
\varphi\left(\xi^{\prime}, x_{3}\right)=\frac{1}{\left|\xi^{\prime}\right|} \operatorname{sh}\left(\left|\xi^{\prime}\right| x_{3}\right) .
$$

Таким образом, частное решение уравнения (7) имеет вид

$$
\widetilde{u}\left(\xi^{\prime}, x_{3}\right)=\frac{1}{\mu\left|\xi^{\prime}\right|} \int_{0}^{x_{3}} \hat{f}\left(\xi^{\prime}, y_{3}\right) \operatorname{sh}\left(\left|\xi^{\prime}\right|\left(x_{3}-y_{3}\right)\right) d y_{3},
$$

а общее решение уравнения (7) -

$$
\begin{aligned}
\widehat{u}\left(\xi^{\prime}, x_{3}\right)= & a\left(\xi^{\prime}\right) e^{-\left|\xi^{\prime}\right| x_{3}}+b\left(\xi^{\prime}\right) e^{\left|\xi^{\prime}\right| x_{3}} \\
& +\frac{e^{\left|\xi^{\prime}\right| x_{3}}}{2 \mu\left|\xi^{\prime}\right|} \int_{0}^{x_{3}} \hat{f}\left(\xi, y_{3}\right) e^{-\left|\xi^{\prime}\right| y_{3}} d y_{3}-\frac{e^{-\left|\xi^{\prime}\right| x_{3}}}{2 \mu\left|\xi^{\prime}\right|} \int_{0}^{x_{3}} \hat{f}\left(\xi^{\prime}, y_{3}\right) e^{\left|\xi^{\prime}\right| y_{3}} d y_{3}
\end{aligned}
$$

где

$$
a\left(\xi^{\prime}\right)=\left\{\begin{array}{ll}
a_{+}\left(\xi^{\prime}\right), & x_{3}>0, \\
a_{-}\left(\xi^{\prime}\right), & x_{3}<0,
\end{array} \quad b\left(\xi^{\prime}\right)=\left\{\begin{array}{ll}
b_{+}\left(\xi^{\prime}\right), & x_{3}>0, \\
b_{-}\left(\xi^{\prime}\right), & x_{3}<0,
\end{array} \quad \mu= \begin{cases}\mu_{+}, & x_{3}>0 \\
\mu_{-}, & x_{3}<0 .\end{cases}\right.\right.
$$

Из условия на бесконечности (4) имеем

$$
\begin{aligned}
& b_{+}\left(\xi^{\prime}\right)=-\frac{1}{2 \mu_{+}\left|\xi^{\prime}\right|} \int_{0}^{+\infty} \hat{f}\left(\xi^{\prime}, y_{3}\right) e^{-\left|\xi^{\prime}\right| y_{3}} d y_{3} \\
& a_{-}\left(\xi^{\prime}\right)=\frac{1}{2 \mu_{-}\left|\xi^{\prime}\right|} \int_{0}^{-\infty} \hat{f}\left(\xi^{\prime}, y_{3}\right) e^{\left|\xi^{\prime}\right| y_{3}} d y_{3} .
\end{aligned}
$$


Из условий сопряжения $(2),(3)$ находим $a_{+}\left(\xi^{\prime}\right)$ и $b_{-}\left(\xi^{\prime}\right)$ :

$$
a_{+}+b_{+}=a_{-}+b_{-}, \quad \mu_{+}\left|\xi^{\prime}\right|\left(-a_{+}+b_{+}\right)=\mu_{-}\left|\xi^{\prime}\right|\left(a_{-}-b_{-}\right)
$$

откуда

$a_{+}=b_{-}+a_{-}-b_{+}, \quad \mu_{+}\left(-a_{+}+b_{+}\right)=\mu_{-}\left(a_{-} b_{-}\right), \quad \mu_{+}\left(2 b_{+}-a_{-}-b_{-}\right)=\mu_{-}\left(a_{-}-b_{-}\right)$,

или

$$
b_{-}\left(\mu_{-}-\mu_{+}\right)=-2 \mu_{+} b_{+}+\left(\mu_{-}+\mu_{+}\right) a_{-} .
$$

Следовательно,

$$
\begin{aligned}
b_{-}\left(\xi^{\prime}\right)= & \frac{1}{\left(\mu_{-}-\mu_{+}\right)\left|\xi^{\prime}\right|} \int_{0}^{+\infty} \hat{f}\left(\xi^{\prime}, y_{3}\right) e^{-\left|\xi^{\prime}\right| y_{3}} d y_{3} \\
& +\frac{\mu_{-}+\mu_{+}}{2 \mu_{-}\left(\mu_{-}-\mu_{+}\right)\left|\xi^{\prime}\right|} \int_{0}^{-\infty} \hat{f}\left(\xi^{\prime}, y_{3}\right) e^{\left|\xi^{\prime}\right| y_{3}} d y_{3}, \\
a_{+}\left(\xi^{\prime}\right)= & \frac{1}{\left(\mu_{-}-\mu_{+}\right)\left|\xi^{\prime}\right|} \int_{0}^{-\infty} \hat{f}\left(\xi^{\prime}, y_{3}\right) e^{\left|\xi^{\prime}\right| y_{3}} d y_{3} \\
& +\frac{\mu_{-}+\mu_{+}}{2 \mu_{+}\left(\mu_{-}-\mu_{+}\right)\left|\xi^{\prime}\right|} \int_{0}^{+\infty} \hat{f}\left(\xi^{\prime}, y_{3}\right) e^{-\left|\xi^{\prime}\right| y_{3}} d y_{3} .
\end{aligned}
$$

Итак, при $x_{3}>0$ решение задачи (1)-(4) в преобразованиях Фурье по $x^{\prime}$ имеет вид (5), а при $x_{3}<0$ соответствующее решение имеет вид (6). Теорема 1 доказана.

\section{2. Оценки решений в полунормах $L_{p}^{2}$. Имеет место}

Теорема 2. Если $f(x) \in \stackrel{\circ}{C}\left(\mathbb{R}^{3}\right), 1<p<\infty$, то полученное в теореме 1 решение задачи (1)-(4) удовлетворяет оченке

$$
\sum_{|\alpha|=2}\left\|D_{x}^{\alpha} u\right\|_{L_{p}\left(\mathbb{R}_{+}^{3}\right)}+\sum_{|\alpha|=2}\left\|D_{x}^{\alpha} u\right\|_{L_{p}\left(\mathbb{R}_{-}^{3}\right)} \leqslant M\|f\|_{L_{p}\left(\mathbb{R}^{3}\right)} .
$$

ДоКАЗАТЕЛЬСТво. Получим оценки в $L_{p}$ для норм вторых производных решения задачи (1)-(4) при $x_{3}>0$. Сделав в интеграле

$$
\int_{0}^{-\infty} \hat{f}\left(\xi^{\prime}, y_{3}\right) e^{\left|\xi^{\prime}\right| y_{3}} d y_{3}
$$

из формулы (5) замену $y_{3}=-y_{3}^{\prime}$, получим при $x_{3}>0$ решение в виде

$$
\begin{aligned}
\widehat{u}\left(\xi^{\prime}, x_{3}\right)= & -\frac{e^{-\left|\xi^{\prime}\right| x_{3}}}{\left(\mu_{-}-\mu_{+}\right)\left|\xi^{\prime}\right|} \int_{0}^{+\infty} \hat{f}\left(\xi^{\prime},-y_{3}\right) e^{-\left|\xi^{\prime}\right| y_{3}} d y_{3} \\
& +\frac{\left(\mu_{-}+\mu_{+}\right) e^{-\left|\xi^{\prime}\right| x_{3}}}{2 \mu_{+}\left|\xi^{\prime}\right|\left(\mu_{-}-\mu_{+}\right)} \int_{0}^{+\infty} \hat{f}\left(\xi^{\prime}, y_{3}\right) e^{-\left|\xi^{\prime}\right| y_{3}} d y_{3} \\
& -\frac{e^{\left|\xi^{\prime}\right| x_{3}}}{2 \mu_{+}\left|\xi^{\prime}\right|} \int_{x_{3}}^{+\infty} \hat{f}\left(\xi^{\prime}, y_{3}\right) e^{-\left|\xi^{\prime}\right| y_{3}} d y_{3}-\frac{e^{-\left|\xi^{\prime}\right| x_{3}}}{2 \mu_{+}\left|\xi^{\prime}\right|} \int_{0}^{x_{3}} \hat{f}\left(\xi^{\prime}, y_{3}\right) e^{\left|\xi^{\prime}\right| y_{3}} d y_{3} .
\end{aligned}
$$


Представим $\widehat{u}\left(\xi^{\prime}, x_{3}\right)$ в виде $\widehat{u}\left(\xi^{\prime}, x_{3}\right)=\widehat{u}_{1}\left(\xi^{\prime}, x_{3}\right)+\widehat{u}_{2}\left(\xi^{\prime}, x_{3}\right)$, где

$$
\begin{aligned}
\widehat{u}_{1}\left(\xi^{\prime}, x_{3}\right)= & -\frac{e^{-\left|\xi^{\prime}\right| x_{3}}}{\left(\mu_{-}-\mu_{+}\right)\left|\xi^{\prime}\right|} \int_{0}^{+\infty} \hat{f}\left(\xi^{\prime},-y_{3}\right) e^{-\left|\xi^{\prime}\right| y_{3}} d y_{3} \\
& +\frac{e^{-\left|\xi^{\prime}\right| x_{3}}\left(\mu_{-}+\mu_{+}\right)}{2 \mu_{+}\left|\xi^{\prime}\right|\left(\mu_{-}-\mu_{+}\right)} \int_{0}^{+\infty} \hat{f}\left(\xi^{\prime}, y_{3}\right) e^{-\left|\xi^{\prime}\right| y_{3}} d y_{3}, \\
\widehat{u}_{2}\left(\xi^{\prime}, x_{3}\right)= & -\frac{1}{2 \mu_{+}\left|\xi^{\prime}\right|} \int_{0}^{+\infty} \hat{f}\left(\xi^{\prime}, y_{3}\right) e^{-\left|\xi^{\prime}\right| \cdot\left|x_{3}-y_{3}\right|} d y_{3} .
\end{aligned}
$$

Получим оценки для вторых производных от $u_{1}\left(\xi^{\prime}, x_{3}\right)$. Умножим $\widehat{u}_{1}\left(\xi^{\prime}, x_{3}\right)$ из $(10)$ на $\left(i \xi_{k}\right)\left(i \xi_{j}\right)$, где $k=1,2, j=1,2$. Тогда

$$
\begin{aligned}
\left(i \xi_{k}\right)\left(i \xi_{j}\right) \widehat{u}_{1}\left(\xi^{\prime}, x_{3}\right)= & -\frac{\left(i \xi_{k}\right)\left(i \xi_{j}\right) e^{-\left|\xi^{\prime}\right| x_{3}}}{\left(\mu_{-}-\mu_{+}\right)\left|\xi^{\prime}\right|} \int_{0}^{x_{3}} \hat{f}\left(\xi^{\prime},-y_{3}\right) e^{-\left|\xi^{\prime}\right| y_{3}} d y_{3} \\
& -\frac{\left(i \xi_{k}\right)\left(i \xi_{j}\right) e^{-\left|\xi^{\prime}\right| x_{3}}}{\left(\mu_{-}-\mu_{+}\right)\left|\xi^{\prime}\right|} \int_{x_{3}}^{+\infty} \hat{f}\left(\xi^{\prime},-y_{3}\right) e^{-\left|\xi^{\prime}\right| y_{3}} d y_{3} \\
& +\frac{\left(i \xi_{k}\right)\left(i \xi_{j}\right) e^{-\left|\xi^{\prime}\right| x_{3}}\left(\mu_{-}+\mu_{+}\right)}{2 \mu_{+}\left|\xi^{\prime}\right|\left(\mu_{-}-\mu_{+}\right)} \int_{0}^{x_{3}} \hat{f}\left(\xi^{\prime}, y_{3}\right) e^{-\left|\xi^{\prime}\right| y_{3}} d y_{3} \\
& +\frac{\left(i \xi_{k}\right)\left(i \xi_{j}\right) e^{-\left|\xi^{\prime}\right| x_{3}}\left(\mu_{-}+\mu_{+}\right)}{2 \mu_{+}\left|\xi^{\prime}\right|\left(\mu_{-}-\mu_{+}\right)} \int_{x_{3}}^{+\infty} \hat{f}\left(\xi^{\prime}, y_{3}\right) e^{-\left|\xi^{\prime}\right| y_{3}} d y_{3} .
\end{aligned}
$$

Введем следующие функции:

$$
\begin{array}{ll}
m_{1}^{k j}\left(\xi^{\prime}, x_{3}, y_{3}\right)=-\frac{\left(i \xi_{k}\right)\left(i \xi_{j}\right) x_{3} e^{-\left|\xi^{\prime}\right|\left(x_{3}+y_{3}\right)}}{\left(\mu_{-}-\mu_{+}\right)\left|\xi^{\prime}\right|}, & x_{3}>y_{3}>0, \\
m_{2}^{k j}\left(\xi^{\prime}, x_{3}, y_{3}\right)=-\frac{\left(i \xi_{k}\right)\left(i \xi_{j}\right) y_{3} e^{-\left|\xi^{\prime}\right|\left(x_{3}+y_{3}\right)}}{\left(\mu_{-}-\mu_{+}\right)\left|\xi^{\prime}\right|}, & y_{3}>x_{3}>0, \\
m_{3}^{k j}\left(\xi^{\prime}, x_{3}, y_{3}\right)=\frac{\left(i \xi_{k}\right)\left(i \xi_{j}\right) x_{3}\left(\mu_{-}+\mu_{+}\right) e^{-\left|\xi^{\prime}\right|\left(x_{3}+y_{3}\right)}}{2 \mu_{+}\left(\mu_{-}-\mu_{+}\right)\left|\xi^{\prime}\right|}, & x_{3}>y_{3}>0, \\
m_{4}^{k j}\left(\xi^{\prime}, x_{3}, y_{3}\right)=\frac{\left(i \xi_{k}\right)\left(i \xi_{j}\right)\left(\mu_{-}+\mu_{+}\right) y_{3} e^{-\left|\xi^{\prime}\right|\left(x_{3}+y_{3}\right)}}{2 \mu_{+}\left(\mu_{-}-\mu_{+}\right)\left|\xi^{\prime}\right|}, & y_{3}>x_{3}>0 .
\end{array}
$$

Все постоянные, не зависяшие от $\xi^{\prime}, x_{3}, y_{3}$, в дальнейшем будем обозначать через $M$.

ПредЛОЖЕниЕ. Функиии $m_{i}^{k j}\left(\xi^{\prime}, x_{3}, y_{3}\right), i=1,2,3,4, k, j=1,2$, являются мультипликаторами из $L_{p}\left(\mathbb{R}^{2}\right)$ в $L_{p}\left(\mathbb{R}^{2}\right), 1<p<\infty$, по $\xi^{\prime}=\left(\xi_{1}, \xi_{2}\right)$, причем константы, которыми они ограничены, не зависят от $x_{3}, y_{3}$.

ДокАЗАТЕЛЬСТво основано на теореме Лизоркина [3] (см. также [7]): пусть функция $m(\xi)$ непрерьвна и ограничена на множестве $\left\{\xi \in \mathbb{R}^{n}, \xi_{j} \neq 0, j=1, \ldots, n\right\}$ вместе со всеми произведениями $\xi^{\beta} D_{\xi}^{\beta} m(\xi), \beta=\left(\beta_{1}, \ldots, \beta_{n}\right)$, где $\beta_{j} \in\{0,1\}$; тогда $m(\xi)$ есть мультипликатор на $L_{p}\left(\mathbb{R}^{n}\right)$, т.е. $m(\xi) \in M_{p}, 1<p<\infty$, при этом норма оператора $T_{m}: L_{p}\left(\mathbb{R}^{n}\right) \rightarrow L_{p}\left(\mathbb{R}^{n}\right), T_{m} u(x)=F^{-1}[m \widehat{u}](x)$ не превосходит $c_{p} \nu$, где

$$
\nu=\sup _{\xi \in \mathbb{R}^{n}}\left|\xi^{\beta} D_{\xi}^{\beta} m(\xi)\right|,
$$


a $c_{p}$ - постоянная, не зависящая от $\nu$.

Докажем предложение для $m_{1}^{k j}\left(\xi^{\prime}, x_{3}, y_{3}\right)$. Очевидно, что функция

$$
m_{1}^{k j}\left(\xi^{\prime}, x_{3}, y_{3}\right)=-\frac{\left(i \xi_{k}\right)\left(i \xi_{j}\right) x_{3} e^{-\left|\xi^{\prime}\right|\left(x_{3}+y_{3}\right)}}{\left(\mu_{-}-\mu_{+}\right)\left|\xi^{\prime}\right|}
$$

где $x_{3}>y_{3}>0,\left|\xi^{\prime}\right|=\sqrt{\xi_{1}^{2}+\xi_{2}^{2}}$, непрерывна и ограничена при $\xi^{\prime}=\left(\xi_{1}, \xi_{2}\right) \in \mathbb{R}^{2}$, $\left|\xi^{\prime}\right| \neq 0$, т.е.

$$
\left|m_{1}^{k j}\left(\xi^{\prime}, x_{3}, y_{3}\right)\right|=\left|\frac{\xi_{k}}{\left|\xi^{\prime}\right|} \frac{\xi_{j}}{\left|\xi^{\prime}\right|} \frac{\left|\xi^{\prime}\right| x_{3} e^{-\left|\xi^{\prime}\right| x_{3} e^{-\left|\xi^{\prime}\right| y_{3}}}}{\mu_{+}+\mu_{-}}\right| \leqslant M .
$$

Имеем

$$
\begin{aligned}
\mid \xi_{1} & \frac{\partial m_{1}^{12}\left(\xi^{\prime}, x_{3}, y_{3}\right)}{\partial \xi_{1}}\left|\leqslant \frac{1}{\mu_{-}-\mu_{+}}\right| \frac{x_{3} \xi_{1} \xi_{2} e^{-\left|\xi^{\prime}\right|\left(x_{3}+y_{3}\right)}}{\left|\xi^{\prime}\right|} \mid \\
& +\left.\frac{1}{\mu_{-}-\mu_{+}}\left|\frac{\xi_{1}^{2}}{\left|\xi^{\prime}\right|^{2}} \frac{\xi_{1}}{\left|\xi^{\prime}\right|} \frac{\xi_{2}}{\left|\xi^{\prime}\right|}\right| \xi^{\prime}\right|^{2}\left(x_{3}^{2} e^{-\left|\xi^{\prime}\right|\left(x_{3}+y_{3}\right)}+x_{3} y_{3} e^{-\left|\xi^{\prime}\right|\left(x_{3}+y_{3}\right)}\right) \mid \\
& +\frac{1}{\mu_{-}-\mu_{+}}\left|\frac{\xi_{1}^{2}}{\left|\xi^{\prime}\right|^{2}} \frac{\xi_{1}}{\left|\xi^{\prime}\right|} \frac{\xi_{2}}{\left|\xi^{\prime}\right|}\left(\left|\xi^{\prime}\right| x_{3} e^{-\left|\xi^{\prime}\right| x_{3}}\right) e^{-\left|\xi^{\prime}\right| y_{3}}\right| \leqslant M .
\end{aligned}
$$

Аналогичным образом доказьваем, что

$$
\left|\xi_{1}^{s_{1}} \xi_{2}^{s_{2}} \frac{\partial^{s_{1}+s_{2}} m_{1}^{k j}}{\partial \xi_{1}^{s_{1}} \partial \xi_{2}^{s_{2}}}\right| \leqslant M
$$

где $s_{l}, l=1,2$, принимают значения 0 или 1 , причем производные

$$
\frac{\partial^{s_{1}+s_{2}} m_{1}^{k j}}{\partial \xi_{1}^{s_{1}} \partial \xi_{2}^{s_{2}}}
$$

непрерьвны при $\xi^{\prime} \in \mathbb{R}^{2},\left|\xi^{\prime}\right| \neq 0$. Тогда по теореме о мультипликаторах $m_{1}^{k j}\left(\xi^{\prime}, x_{3}, y_{3}\right)$ является мультипликатором из $L_{p}$ в $L_{p}$ по $\xi^{\prime}$ с константой, не зависящей от $x_{3}, y_{3}$. Аналогично доказьвается, что $m_{r}^{k j}\left(\xi^{\prime}, x_{3}, y_{3}\right), r=2,3,4, k, j=1,2$, являются мультипликаторами из $L_{p}$ в $L_{p}$ по $\xi^{\prime}$ с константами, не зависящими от $x_{3}, y_{3}$. Предложение доказано.

Положим

$$
\begin{aligned}
& \widehat{\varphi}_{1}^{k j}\left(\xi^{\prime}, x_{3}\right)=\frac{1}{x_{3}} \int_{0}^{x_{3}} \hat{f}\left(\xi^{\prime},-y_{3}\right) m_{1}^{k j}\left(\xi^{\prime}, x_{3}, y_{3}\right) d y_{3} \\
& \widehat{\varphi}_{2}^{k j}\left(\xi^{\prime}, x_{3}\right)=\int_{x_{3}}^{+\infty} \hat{f}\left(\xi^{\prime},-y_{3}\right) m_{2}^{k j}\left(\xi^{\prime}, x_{3}, y_{3}\right) \frac{1}{y_{3}} d y_{3} \\
& \widehat{\varphi}_{3}^{k j}\left(\xi^{\prime}, x_{3}\right)=\frac{1}{x_{3}} \int_{0}^{x_{3}} \hat{f}\left(\xi^{\prime}, y_{3}\right) m_{3}^{k j}\left(\xi^{\prime}, x_{3}, y_{3}\right) d y_{3} \\
& \widehat{\varphi}_{4}^{k j}\left(\xi^{\prime}, x_{3}\right)=\int_{x_{3}}^{+\infty} \hat{f}\left(\xi^{\prime}, y_{3}\right) m_{4}^{k j}\left(\xi^{\prime}, x_{3}, y_{3}\right) \frac{1}{y_{3}} d y_{3} .
\end{aligned}
$$

Известно, что если

$$
\left(T_{m} \widehat{g}\right)\left(x^{\prime}, x_{3}, y_{3}\right)=F_{\xi^{\prime} \rightarrow x^{\prime}}^{-1}\left[m\left(\xi^{\prime}, x_{3}, y_{3}\right) \widehat{g}\left(\xi^{\prime}, y_{3}\right)\right]
$$


то

$$
\left\|\left(T_{m} \widehat{g}\right)\left(x^{\prime}, x_{3}, y_{3}\right)\right\|_{L_{p}\left(\mathbb{R}^{2}\right)} \leqslant M\left\|g\left(x^{\prime}, y_{3}\right)\right\|_{L_{p}\left(\mathbb{R}^{2}\right)}
$$

по определению мультипликатора, здесь и далее норма $L_{p}\left(\mathbb{R}^{2}\right)$ берется по $x^{\prime}$. Применим к (11) обратное преобразование Фурье по $\xi^{\prime}$ :

$$
\begin{aligned}
\varphi_{1}^{k j}\left(x^{\prime}, x_{3}\right) & =\frac{1}{x_{3}} \int_{0}^{x_{3}}\left(T_{m_{1}^{k j}} \hat{f}\right)\left(x^{\prime}, x_{3},-y_{3}\right) d y_{3}, \\
\varphi_{2}^{k j}\left(x^{\prime}, x_{3}\right) & =\int_{x_{3}}^{+\infty}\left(T_{m_{2}^{k j}} \hat{f}\right)\left(x^{\prime}, x_{3},-y_{3}\right) \frac{1}{y_{3}} d y_{3}, \\
\varphi_{3}^{k j}\left(x^{\prime}, x_{3}\right) & =\frac{1}{x_{3}} \int_{0}^{x_{3}}\left(T_{m_{3}^{k j}} \hat{f}\right)\left(x^{\prime}, x_{3}, y_{3}\right) d y_{3}, \\
\varphi_{4}^{k j}\left(x^{\prime}, x_{3}\right) & =\int_{x_{3}}^{+\infty}\left(T_{m_{4}^{k j}} \hat{f}\right)\left(x^{\prime}, x_{3}, y_{3}\right) \frac{1}{y_{3}} d y_{3} .
\end{aligned}
$$

С помощью неравенства Минковского для интегралов из (12) и (13) получаем

$$
\begin{aligned}
&\left\|\varphi_{1}^{k j}\left(x^{\prime}, x_{3}\right)\right\|_{L_{p}\left(\mathbb{R}^{2}\right)} \leqslant M \frac{1}{x_{3}} \int_{0}^{x_{3}}\left(\int_{\mathbb{R}^{2}}\left|f\left(x^{\prime},-y_{3}\right)\right|^{p} d x^{\prime}\right)^{1 / p} d y_{3} \\
&\left\|\varphi_{2}^{k j}\left(x^{\prime}, x_{3}\right)\right\|_{L_{p}\left(\mathbb{R}^{2}\right)} \leqslant M \int_{x_{3}}^{+\infty}\left(\int_{\mathbb{R}^{2}}\left|f\left(x^{\prime},-y_{3}\right)\right|^{p} d x^{\prime}\right)^{1 / p} \frac{1}{y_{3}} d y_{3} \\
&\left\|\varphi_{3}^{k j}\left(x^{\prime}, x_{3}\right)\right\|_{L_{p}\left(\mathbb{R}^{2}\right)} \leqslant M \frac{1}{x_{3}} \int_{0}^{x_{3}}\left(\int_{\mathbb{R}^{2}}\left|f\left(x^{\prime}, y_{3}\right)\right|^{p} d x^{\prime}\right)^{1 / p} d y_{3} \\
&\left\|\varphi_{4}^{k j}\left(x^{\prime}, x_{3}\right)\right\|_{L_{p}\left(\mathbb{R}^{2}\right)} \leqslant M \int_{x_{3}}^{+\infty}\left(\int_{\mathbb{R}^{2}}\left|f\left(x^{\prime}, y_{3}\right)\right|^{p} d x^{\prime}\right)^{1 / p} \frac{1}{y_{3}} d y_{3} .
\end{aligned}
$$

Положим

$$
\begin{array}{ll}
F_{1}\left(y_{3}\right)=\left\|f\left(x^{\prime},-y_{3}\right)\right\|_{L_{p}\left(\mathbb{R}^{2}\right)}, & F_{2}\left(y_{3}\right)=\frac{1}{y_{3}}\left\|f\left(x^{\prime},-y_{3}\right)\right\|_{L_{p}\left(\mathbb{R}^{2}\right)}, \\
F_{3}\left(y_{3}\right)=\left\|f\left(x^{\prime}, y_{3}\right)\right\|_{L_{p}\left(\mathbb{R}^{2}\right)}, & F_{4}\left(y_{3}\right)=\frac{1}{y_{3}}\left\|f\left(x^{\prime}, y_{3}\right)\right\|_{L_{p}\left(\mathbb{R}^{2}\right)}
\end{array}
$$

и рассмотрим операторы Харди $H_{1}$ и $H_{2}$, определяемые для функции $g$, заданной на полуоси $(0,+\infty)$ для любого $x>0$ :

$$
\left(H_{1} g\right)(x):=\frac{1}{x} \int_{0}^{x} g(y) d y, \quad\left(H_{2} g\right)(x):=\frac{1}{x} \int_{x}^{+\infty} g(y) d y .
$$

Тогда в обозначениях (15) неравенства (14) принимают вид

$$
\begin{array}{ll}
\left\|\varphi_{l}^{k j}\left(x^{\prime}, x_{3}\right)\right\|_{L_{p}\left(\mathbb{R}^{2}\right)} \leqslant M\left(H_{1} F_{l}\right)\left(x_{3}\right), & l=1,3, \\
\left\|\varphi_{s}^{k j}\left(x^{\prime}, x_{3}\right)\right\|_{L_{p}\left(\mathbb{R}^{2}\right)} \leqslant M x_{3}\left(H_{2} F_{s}\right)\left(x_{3}\right), & s=2,4 .
\end{array}
$$

Воспользуемся неравенствами Харди (см. [4], [5]): если $\alpha<1 / p^{\prime}, p^{\prime}=p /(p-1)$ $(1<p<+\infty)$, то для любых измеримых на $(0,+\infty)$ функций $f$

$$
\left\|x^{\alpha}\left(H_{1} f\right)(x)\right\|_{L_{p}(0, \infty)} \leqslant\left(\frac{1}{p^{\prime}}-\alpha\right)^{-1}\left\|x^{\alpha} f(x)\right\|_{L_{p}(0, \infty)} ;
$$


если $\alpha>1 / p^{\prime}$, то для любых измеримых на $(0,+\infty)$ функций $f$

$$
\left\|x^{\alpha}\left(H_{2} f\right)(x)\right\|_{L_{p}(0, \infty)} \leqslant\left(\alpha-\frac{1}{p^{\prime}}\right)^{-1}\left\|x^{\alpha} f(x)\right\|_{L_{p}(0, \infty)} .
$$

Тогда

$$
\begin{aligned}
\left\|\left(H_{1} F_{l}\right)\left(x_{3}\right)\right\|_{L_{p}\left(\mathbb{R}_{+}^{1}\right)} & \leqslant \frac{p}{p-1}\left\|F_{l}\left(x_{3}\right)\right\|_{L_{p}\left(\mathbb{R}_{+}^{1}\right)}, & l & =1,3, \\
\left\|x_{3}\left(H_{2} F_{s}\right)\left(x_{3}\right)\right\|_{L_{p}\left(\mathbb{R}_{+}^{1}\right)} & \leqslant p\left\|x_{3} F_{s}\left(x_{3}\right)\right\|_{L_{p}\left(\mathbb{R}_{+}^{1}\right)}, & s & =2,4 .
\end{aligned}
$$

Здесь $\mathbb{R}_{+}^{1}=\left\{x_{3} \mid x_{3}>0\right\}$. Применяя норму пространства $L_{p}\left(\mathbb{R}_{+}^{1}\right)$ по $x_{3}$ к $(16)$ и используя (17), получим

$$
\begin{aligned}
& \left\|\varphi_{l}^{k j}\left(x_{1}, x_{2}, x_{3}\right)\right\|_{L_{p}\left(\mathbb{R}_{+}^{3}\right)} \leqslant M \frac{p}{p-1}\left\|F_{l}\left(y_{3}\right)\right\|_{L_{p}\left(\mathbb{R}_{+}^{1}\right)}, \quad l=1,3, \\
& \left\|\varphi_{s}^{k j}\left(x_{1}, x_{2}, x_{3}\right)\right\|_{L_{p}\left(\mathbb{R}_{+}^{3}\right)} \leqslant M p\left\|y_{3} F_{s}\left(y_{3}\right)\right\|_{L_{p}\left(\mathbb{R}_{+}^{1}\right)}, \quad s=2,4 .
\end{aligned}
$$

Возвращаясь от обозначений (15) к прежним, имеем

$$
\begin{aligned}
& \left\|\varphi_{l}^{k j}\right\|_{L_{p}\left(\mathbb{R}_{+}^{3}\right)} \leqslant M\left(\int_{0}^{+\infty} \int_{\mathbb{R}^{2}}\left|f\left(x^{\prime},-x_{3}\right)\right|^{p} d x^{\prime} d x_{3}\right)^{1 / p}, \quad l=1,2, \\
& \left\|\varphi_{s}^{k j}\right\|_{L_{p}\left(\mathbb{R}_{+}^{3}\right)} \leqslant M\left(\int_{0}^{+\infty} \int_{\mathbb{R}^{2}}\left|f\left(x^{\prime}, x_{3}\right)\right|^{p} d x^{\prime} d x_{3}\right)^{1 / p}, \quad s=3,4 .
\end{aligned}
$$

Заметим, что

$$
\left(\int_{0}^{+\infty} \int_{\mathbb{R}^{2}}\left|f\left(x^{\prime},-x_{3}\right)\right|^{p} d x^{\prime} d x_{3}\right)^{1 / p}=\|f\|_{L_{p}\left(\mathbb{R}_{-}^{3}\right)} .
$$

Таким образом,

$$
\left\|\varphi_{l}^{k j}\right\|_{L_{p}\left(\mathbb{R}_{+}^{3}\right)} \leqslant M\|f\|_{L_{p}\left(\mathbb{R}_{-}^{3}\right)}, \quad l=1,2, \quad\left\|\varphi_{s}^{k j}\right\|_{L_{p}\left(\mathbb{R}_{+}^{3}\right)} \leqslant M\|f\|_{L_{p}\left(\mathbb{R}_{+}^{3}\right)}, \quad s=3,4 .
$$

Tak как

$$
\frac{\partial^{2} u_{1}}{\partial x_{k} \partial x_{j}}=\sum_{s=1}^{4} \varphi_{s}^{k j}, \quad k, j=1,2
$$

получаем

$$
\left\|\frac{\partial^{2} u_{1}}{\partial x_{k} \partial x_{j}}\right\|_{L_{p}\left(\mathbb{R}_{+}^{3}\right)} \leqslant \sum_{s=1}^{4}\left\|\varphi_{s}^{k j}\right\|_{L_{p}\left(\mathbb{R}_{+}^{3}\right)} \leqslant M\left(\|f\|_{L_{p}\left(\mathbb{R}_{-}^{3}\right)}+\|f\|_{L_{p}\left(\mathbb{R}_{+}^{3}\right)}\right),
$$

т.е.

$$
\left\|\frac{\partial^{2} u_{1}}{\partial x_{k} \partial x_{j}}\right\|_{L_{p}\left(\mathbb{R}_{+}^{3}\right)} \leqslant M\|f\|_{L_{p}\left(\mathbb{R}^{3}\right)}, \quad k, j=1,2 .
$$

Аналогично тому, как доказьвались оценки (18), имеем

$$
\left\|\frac{\partial^{2} u_{1}}{\partial x_{3}^{2}}\right\|_{L_{p}\left(\mathbb{R}_{+}^{3}\right)} \leqslant M\|f\|_{L_{p}\left(\mathbb{R}^{3}\right)}, \quad\left\|\frac{\partial^{2} u_{1}}{\partial x_{k} \partial x_{3}}\right\|_{L_{p}\left(\mathbb{R}_{+}^{3}\right)} \leqslant M\|f\|_{L_{p}\left(\mathbb{R}^{3}\right)} .
$$


Таким образом, окончательно получаем

$$
\left\|\frac{\partial^{2} u_{1}}{\partial x_{k} \partial x_{j}}\right\|_{L_{p}\left(\mathbb{R}_{+}^{3}\right)} \leqslant M\|f\|_{L_{p}\left(\mathbb{R}^{3}\right)}, \quad k, j=1,2,3 .
$$

Оценим нормы вторых производных от $u_{2}\left(\xi^{\prime}, x_{3}\right)$. Рассмотрим интеграл (11). Продолжим $\hat{f}\left(\xi^{\prime}, x_{3}\right)$ нулем при $y_{3}<0$. Тогда

$$
\widehat{u}_{2}\left(\xi^{\prime}, x_{3}\right)=-\frac{1}{2 \mu_{+}\left|\xi^{\prime}\right|} \int_{-\infty}^{+\infty} \hat{f}\left(\xi^{\prime}, y_{3}\right) e^{-\left|\xi^{\prime}\right| \cdot\left|x_{3}-y_{3}\right|} d y_{3}=-\frac{1}{2 \mu_{+}\left|\xi^{\prime}\right|} \hat{f}\left(\xi^{\prime}, x_{3}\right) * e^{-\left|\xi^{\prime}\right| \cdot\left|x_{3}\right|} .
$$

Применим к (20) преобразование Фурье по $x_{3}$ :

$$
\begin{aligned}
F_{x_{3} \rightarrow \xi_{3}}\left[\widehat{u}_{2}\left(\xi^{\prime}, x_{3}\right)\right] & =\bar{u}_{2}\left(\xi^{\prime}, \xi_{3}\right)=-\frac{1}{2 \mu_{+}\left|\xi^{\prime}\right|} \bar{f}\left(\xi^{\prime}, \xi_{3}\right) F_{x_{3} \rightarrow \xi_{3}}\left[e^{-\left|\xi^{\prime}\right| \cdot\left|x_{3}\right|}\right] \\
& =-\frac{1}{\mu_{+}} \bar{f}\left(\xi^{\prime}, \xi_{3}\right) \frac{1}{|\xi|^{2}}
\end{aligned}
$$

где $|\xi|^{2}=\xi_{1}^{2}+\xi_{2}^{2}+\xi_{3}^{2}$, так как

$$
F_{x \rightarrow \xi}\left[e^{-a|x|}\right]=\frac{2 a}{\xi^{2}+a^{2}} \quad \text { при } a>0 .
$$

Итак,

$$
F_{x \rightarrow \xi}\left[u_{2}(x)\right]=-\frac{1}{\mu_{+}} \bar{f}(\xi) \frac{1}{|\xi|^{2}}
$$

Заметим, что

$$
F_{x \rightarrow \xi}\left[\frac{\partial^{2} u_{2}\left(x_{1}, x_{2}, x_{3}\right)}{\partial x_{k} \partial x_{j}}\right]=-\frac{\left(i \xi_{k}\right)\left(i \xi_{j}\right)}{\mu_{+}} \bar{f}\left(\xi_{1}, \xi_{2}, \xi_{3}\right) \frac{1}{|\xi|^{2}}, \quad k, j=1,2,3 .
$$

Очевидно, функции

$$
m^{k j}(\xi)=-\frac{\left(i \xi_{k}\right)\left(i \xi_{j}\right)}{\mu_{+}\left(\xi_{1}^{2}+\xi_{2}^{2}+\xi_{3}^{2}\right)}, \quad k, j=1,2,3
$$

являются мультипликаторами из $L_{p}$ в $L_{p}, 1<p<\infty$, по $\xi=\left(\xi_{1}, \xi_{2}, \xi_{3}\right)$. Тогда из $(21)$ получаем

$$
\left\|\frac{\partial^{2} u_{2}\left(x_{1}, u_{2}, u_{3}\right)}{\partial x_{k} \partial x_{j}}\right\|_{L_{p}\left(\mathbb{R}^{3}\right)}=\left\|F_{\xi \rightarrow x}^{-1}\left[m^{k j}(\xi) \bar{f}(\xi)\right]\right\|_{L_{p}\left(\mathbb{R}^{3}\right)} \leqslant\|f\|_{L_{p}\left(\mathbb{R}^{3}\right)} .
$$

Рассмотрим сужение $u_{2}$ на $\mathbb{R}_{+}^{3}=\left\{\left(x_{1}, x_{2}, x_{3}\right) \mid x_{3}>0\right\}$ :

$$
\left\|\frac{\partial^{2} u_{2}\left(x_{1}, u_{2}, u_{3}\right)}{\partial x_{k} \partial x_{j}}\right\|_{L_{p}\left(\mathbb{R}_{+}^{3}\right)} \leqslant M\|f\|_{L_{p}\left(\mathbb{R}^{3}\right)}, \quad k, j=1,2,3 .
$$

Таким образом, из (19), (22) получаем

$$
\left\|\frac{\partial^{2} u\left(x_{1}, u_{2}, u_{3}\right)}{\partial x_{k} \partial x_{j}}\right\|_{L_{p}\left(\mathbb{R}_{+}^{3}\right)} \leqslant M\|f\|_{L_{p}\left(\mathbb{R}^{3}\right)}, \quad k, j=1,2,3 .
$$

Для $x_{3} \in \mathbb{R}_{-}^{3}$ доказательство оценки проводится аналогично, но его можно и не делать, так как при замене $x_{3} \rightarrow-x_{3}^{\prime}$ система (1)-(4) перейдет в себя с точностью до замены $\mu_{-}$ на $\mu_{+}$. Таким образом, имеем (8). Теорема 2 доказана. 
Теорема 3. Для любого $f(x) \in L_{p}\left(\mathbb{R}^{3}\right)$ существует решение задачи (1)-(3) в пространстве $L_{p}^{2}\left(\mathbb{R}_{ \pm}^{3}\right)$.

ДоказАтЕльство. Так как $\stackrel{\circ}{C}^{\infty}\left(\mathbb{R}^{3}\right)$ плотно в $L_{p}\left(\mathbb{R}^{3}\right)$, существование обобщенного решения задачи (1)-(3) в классе $L_{p}^{2}\left(\mathbb{R}_{ \pm}^{3}\right)$ для всех $f(x) \in L_{p}\left(\mathbb{R}^{3}\right)$ доказывается с помощью предельного перехода и оценки (8) из теоремы 2.

3. Ядро оператора, порождаемого задачей (1)-(3).

Теорема 4. Если $f(x) \equiv 0$, то существует нетривиальное решение $и(x) \in$ $L_{p}\left(\mathbb{R}_{ \pm}^{3}\right)$ задачи (1)-(3), являющееся полиномом первой степени.

ДоказАтЕльство. Так как $u(x) \in L_{p}^{2}\left(\mathbb{R}_{ \pm}^{3}\right)$, по теореме Фубини $u\left(x^{\prime}, x_{3}\right) \in L_{p}^{2}\left(\mathbb{R}^{2}\right)$ для почти всех $x_{3} \in \mathbb{R}^{1}$, где $x_{3}$ - параметр. Поэтому преобразование Фурье по $x^{\prime}=$ $\left(x_{1}, x_{2}\right)$ функции $u\left(x^{\prime}, x_{3}\right)$ принадлежит пространству обобщенных функций $S^{\prime}\left(\mathbb{R}^{2}\right)$. Преобразуя по Фурье по $x^{\prime}$ уравнение (1) (c $f(x) \equiv 0$ ) и решая его при $x_{3} \gtrless 0$, получаем общее решение в виде

$$
U_{ \pm}\left(\xi^{\prime}, x_{3}\right)=a_{ \pm}\left(\xi^{\prime}\right) e^{\mp\left|\xi^{\prime}\right| x_{3}}+b_{ \pm}\left(\xi^{\prime}\right) e^{ \pm\left|\xi^{\prime}\right| x_{3}},
$$

где знаки “土” соответствуют $x_{3}>0$ и $x_{3}<0, a_{ \pm}\left(\xi^{\prime}\right), b_{ \pm}\left(\xi^{\prime}\right)$ - обобщенные функции из $S^{\prime}, U_{ \pm}\left(\xi^{\prime}, x_{3}\right)$ - функционалы по $\xi^{\prime}, x_{3}$ - параметр, т.е.

$$
\left\langle U_{ \pm}, \varphi\right\rangle=\left\langle a_{ \pm}\left(\xi^{\prime}\right) e^{\mp\left|\xi^{\prime}\right| x_{3}}, \varphi\left(\xi^{\prime}\right)\right\rangle+\left\langle b_{ \pm}\left(\xi^{\prime}\right) e^{ \pm\left|\xi^{\prime}\right| x_{3}}, \varphi\left(\xi^{\prime}\right)\right\rangle
$$

для всех $\varphi \in S\left(\mathbb{R}^{2}\right), \varphi(0)=0$, в окрестности $\left|\xi^{\prime}\right|=0$. Обозначим это пространство через $\stackrel{\circ}{S}\left(\mathbb{R}^{2}\right)$. Отметим, что последнее требование на пробные функции необходимо для того, чтобы функционалы в (23) были непрерывными. Докажем, что

$$
\left\langle b_{ \pm}\left(\xi^{\prime}\right) e^{ \pm\left|\xi^{\prime}\right| x_{3}}, \varphi\left(\xi^{\prime}\right)\right\rangle=0 .
$$

Для этого рассмотрим действие оператора Лапласа на $u$ во всем $\mathbb{R}^{3}$ с условиями сопряжения $(2),(3)$. Очевидно, существуют производные $\partial u / \partial x_{3}, D_{x^{\prime}}^{\alpha^{\prime}} u,\left|\alpha^{\prime}\right|=2$, в смысле Соболева. Найдем $\partial^{2} u / \partial x_{3}^{2}$ для $x \in \mathbb{R}^{3}$ в смысле обобщенных функций:

$$
\begin{aligned}
\left\langle u, \frac{\partial^{2} \varphi}{\partial x_{3}^{2}}\right\rangle & =-\left\langle\frac{\partial u}{\partial x_{3}}, \frac{\partial \varphi}{\partial x_{3}}\right\rangle=-\int_{-\infty}^{+\infty} \frac{\partial u}{\partial x_{3}} \frac{\partial \varphi}{\partial x_{3}} d x_{3} \\
& =-\int_{-\infty}^{0} \frac{\partial u}{\partial x_{3}} \frac{\partial \varphi}{\partial x_{3}} d x_{3}-\int_{0}^{+\infty} \frac{\partial u}{\partial x_{3}} \frac{\partial \varphi}{\partial x_{3}} d x_{3} \\
& =\int_{-\infty}^{+\infty} \frac{\partial^{2} u}{\partial x_{3}^{2}} \varphi d x_{3}-\left.\frac{\partial u}{\partial x_{3}}\right|_{x_{3}=-0} \varphi\left(x^{\prime}, 0\right)+\left.\frac{\partial u}{\partial x_{3}}\right|_{x_{3}=+0} \varphi\left(x^{\prime}, 0\right) \\
& =\left\langle\left(\left.\frac{\partial u}{\partial x_{3}}\right|_{x_{3}=+0}-\left.\frac{\partial u}{\partial x_{3}}\right|_{x_{3}=-0}\right), \varphi\left(x^{\prime}, 0\right)\right\rangle+\int_{-\infty}^{+\infty} \frac{\partial^{2} u}{\partial x_{3}^{2}} \varphi d x_{3} \\
& =\left\langle h\left(x^{\prime}\right) \delta\left(x_{3}\right), \varphi\left(x^{\prime}\right)\right\rangle+\left[\frac{\partial^{2} u}{\partial x_{3}^{2}}\right]
\end{aligned}
$$

для всех $\varphi \in S\left(\mathbb{R}^{3}\right)$. Таким образом, для $x \in \mathbb{R}^{3}$ в смысле обобщенных функций имеем

$$
\Delta u=h\left(x^{\prime}\right) \delta\left(x_{3}\right)
$$


где

$$
h\left(x^{\prime}\right)=\left.\frac{\partial u\left(x^{\prime}, x_{3}\right)}{\partial x_{3}}\right|_{x_{3}=+0}-\left.\frac{\partial u\left(x^{\prime}, x_{3}\right)}{\partial x_{3}}\right|_{x_{3}=-0} .
$$

Преобразуем по Фурье по $x \in \mathbb{R}^{3}$ уравнение $(24):|\xi|^{2} \widehat{u}(\xi)=\widehat{h}\left(\xi^{\prime}\right)$. Найдем обратное преобразование $\Phi$ урье по $x_{3}$ :

$$
\widehat{u}\left(\xi^{\prime}, x_{3}\right)=F_{\xi_{3} \rightarrow x_{3}}^{-1} \frac{\widehat{h}\left(\xi^{\prime}\right)}{\left|\xi^{\prime}\right|^{2}+\xi_{3}^{2}}=\widehat{h}\left(\xi^{\prime}\right) \frac{\pi}{\left|\xi^{\prime}\right|} e^{-\left|\xi^{\prime}\right| \cdot\left|x_{3}\right|},
$$

раскрьвая модуль $\left|x_{3}\right|$ в зависимости от знака $x_{3}$. Записьвая $(25)$ в виде функционала и сравнивая с $(23)$, получаем $b_{ \pm}\left(\xi^{\prime}\right) \equiv 0$. Таким образом, общее решение в смысле $S^{\prime}$ задачи (1)-(3) имеет вид

$$
U\left(\xi^{\prime}, x_{3}\right)= \begin{cases}a_{+}\left(\xi^{\prime}\right) e^{-x_{3}\left|\xi^{\prime}\right|}, & x_{3}>0 \\ a_{-}\left(\xi^{\prime}\right) e^{x_{3}\left|\xi^{\prime}\right|}, & x_{3}<0 .\end{cases}
$$

Найдем $a_{+}\left(\xi^{\prime}\right)$ и $a_{-}\left(\xi^{\prime}\right)$ из условий сопряжения. Имеем $\left\langle a_{+}\left(\xi^{\prime}\right), \varphi\left(\xi^{\prime}\right)\right\rangle=\left\langle a_{-}\left(\xi^{\prime}\right), \varphi\left(\xi^{\prime}\right)\right\rangle$ для всех $\varphi \in \stackrel{\circ}{S}\left(\mathbb{R}^{2}\right)$, откуда следует, что $a_{+}\left(\xi^{\prime}\right)=a_{-}\left(\xi^{\prime}\right)$. Из второго условия сопряжения (3) находим $\mu_{+}\left|\xi^{\prime}\right| a_{+}\left(\xi^{\prime}\right)=\mu_{-}\left|\xi^{\prime}\right| a_{-}\left(\xi^{\prime}\right)$, причем все равенства вьполняются в смысле $S^{\prime}$ для всех $\varphi \in \stackrel{\circ}{S}\left(\mathbb{R}^{2}\right)$. Из последних двух равенств, так как $\mu_{+} \neq \mu_{-}$, получаем $\left\langle a_{+}\left(\xi^{\prime}\right), \varphi\left(\xi^{\prime}\right)\right\rangle=0$ для всех $\varphi \in \stackrel{\circ}{S}\left(\mathbb{R}^{2}\right)$, откуда следует, что $\operatorname{supp} a_{ \pm}\left(\xi^{\prime}\right)=\{0\}$, т.е.

$$
a_{ \pm}\left(\xi^{\prime}\right)=\sum_{\left|\alpha^{\prime}\right| \leqslant N} c_{k} D_{\xi^{\prime}}^{\alpha^{\prime}} \delta\left(\xi^{\prime}\right) .
$$

Но решение принадлежит $L_{p}^{2}\left(\mathbb{R}_{ \pm}^{3}\right)$, значит нетривиальное решение задачи $(1)-(3)$ с $f \equiv 0$ имеет вид $u(x)=a_{1}\left(x_{3}\right) x_{1}+a_{2}\left(x_{3}\right) x_{2}+a_{3}\left(x_{3}\right)$ при $x_{3} \gtrless 0$. Так как $\Delta u=0$, то $a_{1}^{\prime \prime}\left(x_{3}\right) x_{1}+a_{2}^{\prime \prime}\left(x_{3}\right) x_{2}+a_{3}^{\prime \prime}=0$, т.е. $a_{k}\left(x_{3}\right)$ должны быть многочленами первого порядка:

$$
u(x)=\left(\alpha_{1} x_{3}+\alpha_{0}\right) x_{1}+\left(\beta_{1} x_{3}+\beta_{0}\right) x_{2}+\gamma_{1} x_{3}+\gamma_{0} .
$$

Ho

$$
\frac{\partial^{2} u}{\partial x_{1} \partial x_{3}}=\alpha_{1}, \quad \frac{\partial^{2} u}{\partial x_{2} \partial x_{3}}=\beta_{1}
$$

а поскольку $u(x) \in L_{p}^{2}\left(\mathbb{R}_{ \pm}^{2}\right)$, то $\alpha_{1}=\alpha_{2}=0$.

Таким образом, при $x_{3} \gtrless 0$ имеем $u_{ \pm}(x)=\alpha_{0}^{ \pm} x_{1}+\beta_{0}^{ \pm} x_{2}+\gamma_{1}^{ \pm} x_{3}+\gamma_{0}^{ \pm}$. Коэффициенты этого полинома должны удовлетворять условиям сопряжения:

$$
\left.u\right|_{x_{3}=+0}=\left.u\right|_{x_{3}=-0},
$$

откуда $\alpha_{0}^{+} x_{1}+\beta_{0}^{+} x_{2}+\gamma_{0}^{+}=\alpha_{0}^{-} x_{1}+\beta_{0}^{-} x_{2}+\gamma_{0}^{-}$, т.е. $\alpha_{0}^{+}=\alpha_{0}^{-}=\alpha_{0}, \beta_{0}^{+}=\beta_{0}^{-}=\beta_{0}$, $\gamma_{0}^{+}=\gamma_{0}^{-}=\gamma_{0}$;

$$
\left.\mu_{+} \frac{\partial u}{\partial n}\right|_{x_{3}=+0}=\left.\mu_{-} \frac{\partial u}{\partial n}\right|_{x_{3}=-0},
$$

откуда $\mu_{+} \gamma_{1}^{+}=-\mu_{-} \gamma_{1}^{-}$. Таким образом, ядро дифференциального оператора, порождаемого задачей $(1)-(3)$, с областью определения $L_{p}^{2}\left(\mathbb{R}_{ \pm}^{3}\right)$ имеет вид

$$
u_{ \pm}(x)=\alpha_{0} x_{1}+\beta_{0} x_{2}+\gamma_{1}^{ \pm} x_{3}+\gamma_{0},
$$

где $\alpha_{0}, \beta_{0}, \gamma_{0}, \gamma_{1}^{ \pm}$- произвольные постоянные. Теорема 4 доказана. 
СлеДСтвИЕ 1. Размерность ядра дифференииального оператора, порождаемого задачей (1)-(3), с областью определения $L_{p}^{2}\left(\mathbb{R}_{ \pm}^{3}\right)$ и областью значений $L_{p}\left(\mathbb{R}^{3}\right)$ равна 1.

СлЕДСТВИЕ 2. Если от решения $u(x) \in L_{p}^{2}\left(\mathbb{R}_{ \pm}^{3}\right)$ потребовать выполнения условия убывания (4) при $\left|x_{3}\right| \rightarrow \infty$, то решение задачи (1)-(4) будет единственным.

Однако, вопрос о существовании такого решения для $f(x) \in L_{p}\left(\mathbb{R}^{3}\right)$ остается открытым; для $f(x) \in \stackrel{\circ}{C}\left(\mathbb{R}^{3}\right)$ оно построено в явном виде.

\section{СПИСОК ЦИТИРОВАННОЙ ЛИТЕРАТУРЫ}

[1] Масленникова В.Н., Боговский М. Е. Гидродинамический подход к моделированию динамики морских волн, создаваемых ураганом // Proceedigs of the 9th International Conference "Computational Modelling and Computing in Phisics". Дубна, 1997. C. 352-359.

[2] Maslennikova V. N., Bogovskii M. E. Generalized solution in the large for the NavierStokes system with a free surface separating two fluids // International Conference "Nonlinear Partial Differential Equations". Abstracts. Kiev, 1997. P. 16.

[3] Лизоркин П.И. Обобщенное лиувиллевское дифференцирование и метод мултипликаторов в теории вложений классов дифференцируемых функций // Тр. МИАН. 1969. Т. 105. C. $89-167$.

[4] Буренков В.И. Функциональные пространства. Основные интегральные неравенства, связанные с пространствами $L_{p}$. М.: Изд. УДН, 1989.

[5] Стейн И.М. Сингулярные интегралы и дифференциальные свойства функций. М.: Мир, 1973.

[6] Владимиров В. С. Обобщенные функции в математической физике. М.: Наука, 1976.

[7] Демиденко Г. В., Успенский С. В. Уравнения и системы, не разрешенные относительно старшей производной. Новосибирск: Научная книга, 1998.

Российский университет Дружбы народов 